\title{
Photon Migration and Spatial Resolution in Spectroscopic Imaging
}

\author{
N Everall*, T Hahn**, P Matousek***, A Parker*** and M Towrie*** \\ *ICI Measurement Science Group, Wilton, Redcar, TS10 4RF, (UK) \\ ** National Starch and Chemical, 10 Finderne Ave., Bridgewater, NJ (USA) \\ *** Rutherford Appleton Laboratory, Chilton, Didcot, Oxford, OX1 0QX (UK)
}

Photon migration is the term commonly used to describe the process by which photons propagate through turbid media. The process involves photons travelling between scattering centres, whereupon they are scattered either randomly (diffusive) or with a forward-biased angular distribution (ballistic). The distance between scattering events is termed the scattering length. A correct understanding and modelling of this process is required in order to understand and model light transport through systems such as colloidal dispersions, slurries and biological tissues, which in turn is important for particle sizing and imaging the structure of living tissues. Considerable effort has been expended on measuring and modelling these photon transport processes in the time and frequency regimes [1-3].

Recently we reported the observation of photon migration in time-resolved Raman spectroscopy, where it was shown that Raman and Tyndall photons are re-emitted on different timescales after the incidence of a picosecond laser pulse on a powdered sample [4]. It was shown how Raman and Tyndall photons traversed path lengths of several centimetres in powdered samples prior to reemission and detection. Given this observation, it is important to consider how this might affect the spatial resolution of spectroscopic imaging experiments. In other words, even though photons are focused to just a few microns on a sample surface, might they sample regions quite distant from the focus prior to being re-emitted and detected? This is particularly important in global imaging experiments, where a photon might emerge some distance from its entrance point and still be observed, thereby degrading the spatial resolution (Fig 1).

To investigate this phenomenon, we have developed simple analytical and Monte Carlo models which describe the propagation of light under elastic and inelastic scattering conditions, and used these to investigate the spatial resolution of micro-spectroscopy and global imaging instruments. The main findings for a typical configuration with solely diffusive scatter were:

- Only photons with paths confined near the sample surface tend to be re-emitted and detected, so the average sampling depth tends to be $\sim 1 / 10$ the total path length (and $<20 \%$ of the typical scattering length).

- Raman photons, due to their stochastic generation mechanism, tend on average to be generated at points further from the origin than the average (observed) Tyndall photon distribution hence the resolution of Raman imaging should be poorer than that of "white light" imaging.

- In point microspectroscopy, the spatial resolution is controlled by the ratio of the collection "aperture" to the scattering length - hence the resolution of point microscopy is not badly degraded by photon migration, provided the scattering length greatly exceeds the collection aperture. 
- In global spectroscopic imaging, the lateral and depth resolution is ultimately determined by the scattering length. For example, in Tyndall scattering the computed lateral resolution was about $60 \%$ of the scattering length, whereas the lateral resolution computed for Raman scattering was almost twice the scattering length.

- If a time resolved experiment is performed, one can control the average sampling depth by varying the collection time, thus some degree of depth profiling should be achievable.

In short, the main conclusion from our work is that in global imaging systems, the spatial resolution is determined mainly by the scattering properties of the sample rather than the optical properties of the instrument. Therefore a good understanding of the optics of the sample is very important in interpreting spectroscopic images from turbid systems. The presentation will demonstrate these results using a range of configurations, and will also discuss the influence of ballistic photon scattering.

References

1 R. F. Bonner et al., J Opt. Soc. Am. A 4 (1987) 423.

2 S. M. Richter and E. M. Sevick-Muraca, Colloids Surf. A 172 (2000) 163.

3 P. P. Ho et al., Appl. Opt. 28 (1989) 2304.

4 N. Everall et al., Appl. Spectrosc. 55 (2001) 1701.

\section{Incident photon Emergent photon}

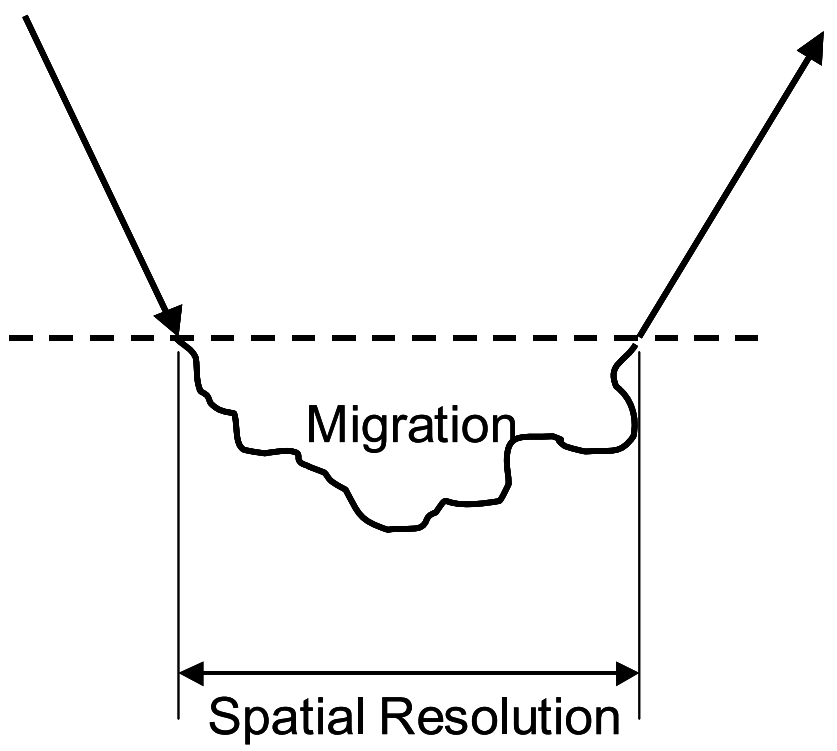

Figure 1 Schematic showing how in a turbid system, photon migration can ultimately control the effective spatial resolution of a global imaging experiment. Photons incident at one point can emerge at a distant position having interacted with material deep in the sample. This can complicate the interpretation of spectroscopic images 